\title{
Educação Permanente: Estratégia Resolutiva na Enfermagem
}

\author{
Ana Paula Agostinho Alencar ${ }^{1 .}$; Fernando Luiz Affonso Fonseca ${ }^{2 ;}$ Marisângela da Costa Silva ; Andrea Maria \\ Casado Marques $^{4}$; Petrúcya Frazão Lira ${ }^{5}$; Camila Macêdo de Figueiredo ${ }^{6}$; Samyra Paula Lustoza Xavier ${ }^{7}$
}

\begin{abstract}
Resumo: O Objetivo do presente estudo é descrever em forma de relato de experiência, um projeto de intervenção educação permanente em saúde, implementado em uma Policlínica. Método: Trata-se de uma pesquisa descritiva, exploratória com abordagem quantitativa, do tipo relato de experiência realizada na policlínica Barbara Pereira de Alencar. De Fevereiro a outubro de 2015. Publico alvo funcionários do estabelecimento. Resultados: realização das atividades de 23/03/15 à 17/08/2015 temas acolhimento, aula expositiva/oficina. Ética no serviço profissional, roda de conversa. Técnicas de curativo e tipos de cobertura, aula expositiva. Biossegurança, aula expositiva e prática. Centro cirúrgico, aula expositiva e prática. Esterilização, oficina e prática no setor. Discussão: Os modelos assistenciais pautados pela integralidade devem adotar formas ampliadas de captação das necessidades de saúde, criando possibilidades para atender não somente aquilo que está programado, ou que é identificado como relevante pelos saberes tradicional. Conclusão: A Educação Permanente essencial, partindo do ponto que a mesma deve ser realizada de forma dinâmica, envolvente, técnica com envolvimento de grupos buscando melhorias no serviço e trabalhando a necessidade de cada um, ou seja focado na coletividade.
\end{abstract}

Palavras-chave: Educação Permanente. Enfermagem. Estratégia resolutiva.

\section{Continuing Education: Strategy Resolutive in Nursing}

\begin{abstract}
The purpose of this study is describe in the form of experience report the intervention project permanent health education implemented in a polyclinic. Method: This is a descriptive, exploratory study with a quantitative approach, the type experience report conducted in polyclinic Barbara Pereira de Alencar. From February to October 2015. Public targeted employees of the establishment. Results: perform activities of 23/03/15 to 17/08/2015 themes host exhibition / workshop class. Ethics in professional service, conversation wheel. healing techniques and types of coverage, lecture. Biosecurity, expository and practical class. surgical center, lecture and practice. Sterilization workshop and practice in the industry. Discussion: The care models guided by integrity should adopt extended forms of funding health needs, creating opportunities to meet not only what is programmed, or is identified as relevant for traditional knowledge. Conclusion: Permanent Education essential from the point that it must be performed dynamically, engaging, involving technical groups seeking improvements in service and working the needs of each one, that is focused on the community.
\end{abstract}

Keywords: Continuing Education. Nursing. Solving strategy.

\footnotetext{
${ }^{1}$ Bacharel em Enfermagem pela Universidade Regional do Cariri-URCA; Pós graduada em Saúde da Família e Prática Docente no Ensino Superior, ambas pela faculdade Integrada de Patos FIP. Especialista em Sistema de Regulação no SUS, pelo hospital sírio libanês. Especialista em Educação Permanente pela Universidade Federal do Rio Grande do Sul. Especializanda em Gerenciamento de risco pelo Hospital Sírio Libanês. Mestrado em ciências da saúde Faculdade de Medicina do ABC Paulista (2013- 2015). E-mail: enfanapaula@hotmail.com;

${ }^{2}$ Pós doutorado pelo Instituto Israelita de Ensino e Pesquisa (IIEP-HIAE) concluído em 2009 e pós doutorado pelo Roche Center for Medical Genomics (Basel-Suiça) Hoffman - La Roche concluído em 2006 e o Doutorado em Medicina (Hematologia) pela Faculdade de Medicina da Universidade de São Paulo, é Farmacêutico-Bioquímico com habilitação em análises clínicas pela Universidade Paulista. Atualmente é Professor Adjunto IV da Universidade Federal de São Paulo do Campus Diadema.

${ }^{3}$ Especialização pela Faculdade de Medicina de Juazeiro do Norte . Atualmente é Coordenador da Atenção Básica da Prefeitura Municipal de Santana do Cariri.

${ }^{4}$ Graduação em Enfermagem e Obstetrícia pela Universidade Estadual da Paraíba. Enfermeira da Prefeitura Municipal de Santana do Cariri;

${ }^{5}$ Graduação em Enfermagem pela Universidade Estadual Vale do Acaraú , Especialista em Biologia pela Faculdade do Juazeiro do Norte, Especialista em Nefrologia pela Universidade Regional Vale do Acaraú, Especialista em Educação na Saúde para Preceptores do SUS pelo Hospital Sírio Libanês, Especializanda em Gestão da Vigilância em saúde pelo Sírio Libanês, Especializanda em Saúde da Família pela Universidade de Brasília e Mestranda em Ciências da Educação pela ISEL. Docente da Faculdade de Juazeiro do Norte-FJN.

${ }^{6}$ Graduação em Enfermagem pela Universidade de Pernambuco e Especialização em Especialização em Saúde da Família pela Universidade Regional do Cariri . Professor Assistente na Faculdade de Juazeiro do Norte.

${ }^{7}$ Bacharel em Enfermagem pela Universidade Regional do Cariri - URCA. Professora do Instituto Centro de Ensino Tecnológico (CENTEC) - Curso Técnico de Enfermagem. Cursando Pós-Graduação Lato Sensu em Enfermagem em Emergência e Cuidados Intensivos no Centro de Educação São Camilo.
} 


\section{Introdução}

Não é novidade que á saúde anda por passos rasos, lentos em busca de melhorias e satisfação de quem a utiliza. Esta dificuldade pode estar ligada a fatos históricos, quem sabe no evento de que a saúde nunca foi ou é tema essencial de preocupação dos governantes do país? Quem conhece todo inicio sabe que não, políticas e resolutividades surgiam a partir de problemas identificados e necessidades de resolução imediata.

Tudo teve inicio logo com a colonização, a partir de população em aglomerados, o próprio crescimento populacional e surgimento de diversas doenças infecciosas as quais se procuravam uma cura, ao mesmo tempo em que tudo se caracterizava como fora de controle surgia à ideia de saúde coletiva, prevenção, coletividade, controle aparecendo como marco histórico o Oswaldo Cruz.

É interessante perceber o contexto histórico em que a proposta de educação permanente surge na década de 80. Nessa época, no Brasil e em contextos semelhantes de outros países da América Latina, era o momento em que se iniciava o processo de democratização política no país, sendo, nessa década, aprovada a nova Constituição e as Leis do SUS, que garantia direitos a população, relacionado à saúde (SANTOS, TENÓRIO; KICH, 2011).

A defesa do trabalho preventivo permanece até nos dias atuais mas, resta a reflexão sobre como este trabalho é realizado e consequentemente a indagação: $\mathrm{O}$ trabalho preventivo é acreditado e resolutivo? A lei 8080 junto a CF de 1988 estabelece saúde como direito de todos e dever do estado, todos com direito as condições para a promoção, proteção e recuperação da saúde, a organização e o funcionamento dos serviços (BRASIL, 1990).

Para colocar em pratica uma estratégia é necessário planejamento e obvio a estratégia, e qual tática de se trabalhar prevenção, promoção da saúde? Uma das estratégias é a educação permanente em saúde que se conceitua de acordo com organização mundial da saúde em 1982 sendo um processo que inclui as experiências posteriores ao adestramento inicial, que ajudam o pessoal a aprender competências importantes para o seu trabalho. Vale ressaltar a diferença existente entre educação permanente e educação continuada. Em 1978, a Organização Pan Americana da Saúde (OPS) conceitua a Educação Continuada como um processo permanente que se inicia após a formação básica e tem como intuito atualizar e melhorar a capacidade de uma pessoa ou grupo, frente à evolução técnico-científica e às necessidades sociais Lisiane (FLORES; ILHA, 2001).

Segue quadro simplificando as principais diferenças entre Educação continuada e Educação Permanente em saúde (SANTOS;TENÓRIO;KICH, 2011). 
Id on Line Revista Multidisciplinar e de Psicologia

Id on Line Multidisciplinary and Psychology Journal

\begin{tabular}{|l|l|}
\hline EDUCAÇÃO CONTINUADA & EDUCAÇÃO PERMANENTE \\
\hline Âmbito individual & Âmbito coletivo \\
\hline Atualizações técnicas & Formação integral continuada \\
\hline Tema de capacitação elaborado previamente & $\begin{array}{l}\text { O tema surge na necessidade de resolver um } \\
\text { problema, identificado no cotidiano do trabalho, na } \\
\text { gestão e no controle social }\end{array}$ \\
\hline $\begin{array}{l}\text { O objetivo central da aprendizagem são os temas } \\
\text { técnicos e científicos }\end{array}$ & $\begin{array}{l}\text { O objetivo central da aprendizagem é o processo } \\
\text { de trabalho }\end{array}$ \\
\hline Capacitações pontuais & $\begin{array}{l}\text { Educação em serviço articulada a mudanças de } \\
\text { práticas. }\end{array}$ \\
\hline
\end{tabular}

Baseado na $1^{\text {a }}$ Portaria GM/MS n 1.996 , de 20 de agosto de 2007 que dispõe sobre as diretrizes para a implementação da Política Nacional de Educação Permanente em Saúde e dá outras providências (BRASIL, 2006).

A educação permanente não nega a importância do conhecimento científico, mas busca articular esse tipo de saber ao conhecimento na ação e a outros aspectos que possam potencializar a ação pedagógica na perspectiva da melhoria dos serviços de saúde. Como ação educativa, a educação permanente deve ser diferenciada de outros processos educacionais que comumente observamos acontecer na área da saúde. Uma dessas diferenças diz respeito à educação continuada, cujo foco é a aquisição de novos conhecimentos técnicos como forma de atualização e acompanhamento dos avanços científicos das áreas (SANTOS; TENÓRIO,KICH,2011, BRASIL, 2006).

A Política Nacional de Educação Permanente em Saúde deve considerar as especificidades regionais, a superação das desigualdades regionais, as necessidades de formação e desenvolvimento para o trabalho em saúde e a capacidade já instalada de oferta institucional de ações formais de educação na saúde (BRASIL, 2006).

Adotar a educação permanente como eixo para a educação na saúde não implica negar por completo a atualização científica, mas busca subordinar essa finalidade ao contexto das instituições de saúde e às necessidades dos serviços (SANTOS; TENÓRIO; KICH, 2011).

Então surgi às perguntas existe efeito a utilização da educação em saúde? A mesma é realizada nas instituições é dada importância desta atividade pelos profissionais na pratica profissional do dia a dia. O objetivo do trabalho foi à realização de atividades de educação permanente no serviço de saúde de atuação, de forma mensal, fechando conclusão por semestre diante de temáticas escolhidos pelos funcionários.

Diante de experiências profissionais, convívio diário com grupos de pessoas as quais são cobrados atividades descritas nos próprios códigos de ética e que não são realizadas, em busca de mudança, aperfeiçoamento e inovação surgiram o interesse em trabalhar com a temática. Junto ao desejo de deixar um produto de trabalho na instituição de atuação e começar a melhoria, deu se a 
escolha em trabalhar com atividades relacionadas à educação permanente na própria unidade de trabalho com temáticas escolhidas pelos funcionários e colegas de trabalho.

Espera se com a conclusão de todas estas atividades uma melhoria no serviço não apenas de forma técnica nas temáticas trabalhadas, mas na interação interpessoal, no convívio e principalmente envolvimento usuário e funcionários do serviço. Busca se uma qualidade de serviço.

\section{Método}

Trata - se de uma pesquisa descritiva, exploratória com abordagem quantitativa, do tipo relato de experiência realizada na policlínica Barbara Pereira de Alencar, serviço da atenção secundaria a saúde. No período fevereiro a outubro de 2015.

O relato foi baseado em Estudo realizado a partir da organização de um projeto de intervenção, ou seja, caracterizado como uma pesquisa ação que segundo Gil (GIL, 2010) vem emergindo como uma metodologia de intervenção, desenvolvimento e mudanças no âmbito de grupos, organizações e comunidades. O propósito é de proporcionar a aquisição de conhecimentos claros, precisos e objetivos.

Com atividades distribuídas de forma mensal a partir de cronograma pré elaborado pelas enfermeiras, depois de um pré diagnóstico feito com todos os profissionais da instituição para escolha das necessidades, outro ponto levado em consideração para escolha das temáticas foi a observação constante por semanas do trabalho desenvolvido pelos funcionários, detectando desde então as prioridades.

O Público alvo foram todos os funcionários do estabelecimento, adequando a categoria (a) ao tema trabalhado no momento. Em média participaram 20 profissionais em cada atividade, dentre eles 16 mulheres e quatro homens estes pertencentes a equipe de serviços gerais e recepção. Não foi realizado uma caracterização especifica da amostra em relação a idade, escolaridade dentre outros fatores pois, o principio de essência era que todos participassem das atividades, e que todos seriam funcionários da instituição não existindo nenhum critério de exclusão.

\section{Resultados}

Os resultados serão apresentados em quadro para melhor apreciação dos leitores. Quadro este com demonstração da data e horário, temática trabalhada, estratégia utilizada e público participativo.

Todas as atividades foram realizadas na sede da Policlínica Bárbara Pereira de Alencar na cidade de Campos Sales - CE. 
Id on Line Revista Multidisciplinar e de Psicologia

Id on Line Multidisciplinary and Psychology Journal

As capacitações foram realizadas Pelas enfermeiras do serviço, em algumas capacitações permaneciam as duas, motivo escala de trabalho.

Segue distribuídos no quadro abaixo as atividades realizadas segundo cronograma traçado.

\begin{tabular}{|c|c|c|c|}
\hline DATA/HORÁRIO & TEMA & ESTRATÉGIA & $\begin{array}{c}\text { PÚBLICO } \\
\text { PARTICIPANTE }\end{array}$ \\
\hline $\begin{array}{l}\text { 23/03/2015 } \\
\text { Às 8:30h }\end{array}$ & ACOLHIMENTO & $\begin{array}{c}\text { AULA } \\
\text { EXPOSITIVA/OFICINA } \\
\text { COM ESTUDOS DE CASO }\end{array}$ & $\begin{array}{c}\text { TECNICOS DE } \\
\text { ENFERMAGEM, } \\
\text { RECEPÇÃO, } \\
\text { FARMACEUTICO E } \\
\text { SERVIÇOS GERAIS }\end{array}$ \\
\hline $\begin{array}{l}06 / 04 / 2015 \\
\text { Às } 8: 30 \mathrm{~h} \\
16 / 04 / 2015 \\
\text { Às } 14: 00 \mathrm{~h}\end{array}$ & $\begin{array}{c}\text { ÉTICA NO } \\
\text { SERVIÇO } \\
\text { PROFICIONAL }\end{array}$ & $\begin{array}{l}\text { RODA DE CONVERSA. } \\
\text { INTRODUÇÃO DE FORMA } \\
\text { EXPOSITIVA. }\end{array}$ & $\begin{array}{l}\text { TODOS OS } \\
\text { PROFISSIONAIS DO } \\
\text { SERVIÇO. }\end{array}$ \\
\hline $\begin{array}{l}25 / 05 / 2015 \\
\text { Às } 8: 30 \mathrm{~h}\end{array}$ & $\begin{array}{l}\text { TÉCNICAS DE } \\
\text { CURATIVO E } \\
\text { TIPOS DE } \\
\text { COBERTURA }\end{array}$ & $\begin{array}{l}\text { USO DE SLAIDS PARA } \\
\text { INTRODUÇÃO } \\
\text { EXPOSITIVA. } \\
\text { DEMOSTRAÇÃO DOS } \\
\text { MATERIAIS DE FORMA } \\
\text { PRÁTICA. PRÁTICA NO } \\
\text { SERVIÇO COM } \\
\text { SUPERVISÃO DO } \\
\text { ENFERMEIRO }\end{array}$ & $\begin{array}{l}\text { TÉCNICOS DE } \\
\text { ENFERMAGEM }\end{array}$ \\
\hline $\begin{array}{l}29 / 06 / 2015 \\
\text { ÀS } 14: 30\end{array}$ & BIOSSEGURANÇA & $\begin{array}{c}\text { AULA EXPOSITIVA. } \\
\text { DEMONSTRAÇÃO DOS } \\
\text { MATERIAIS E PRÁTICA. }\end{array}$ & $\begin{array}{c}\text { TÉCNICOS DE } \\
\text { ENFERMAGEM, } \\
\text { LABORATÓRIO E } \\
\text { SERVIÇOS GERAIS. }\end{array}$ \\
\hline $07 / 07 / 2015$ & $\begin{array}{l}\text { CENTRO } \\
\text { CIRURGICO }\end{array}$ & $\begin{array}{c}\text { AULA EXPOSITIVA. } \\
\text { PRÁTICA NO CENTRO } \\
\text { CIRURGICO } \\
\text { SUPERVISIONADO PELO } \\
\text { ENFERMEIRO }\end{array}$ & $\begin{array}{c}\text { TÉCNICOS DE } \\
\text { ENFERMAGEM }\end{array}$ \\
\hline $17 / 08 / 2015$ & ESTERILIZAÇÃO & $\begin{array}{l}\text { TÉORIA EM FORMATO } \\
\text { OFICINA. PRÁTICA NO } \\
\text { SETOR. }\end{array}$ & $\begin{array}{c}\text { TECNICO DE } \\
\text { ENFERMAGEM }\end{array}$ \\
\hline
\end{tabular}

\section{Discussão}

Foi visto no decorrer de cada atividade que a educação permanece faz essencial dentro do planejamento do serviço de saúde e fica na responsabilidade do enfermeiro em trabalhar, acompanhar, incentivar e avaliar esta pratica.

As atividades foram sendo realizadas de acordo com cronograma, necessidade e ideias dos participantes. $\mathrm{O}$ intuito era o envolvimento dos participantes e posteriormente a implantação do aprendizado no serviço. A primeira oficina, acolhimento foi um sucesso realizada em forma de oficina onde foram entregues estudos de caso com situações problemas 
Id on Line Revista Multidisciplinar e de Psicologia

Id on Line Multidisciplinary and Psychology Journal

de mau acolhimento que já tinham acontecido no próprio estabelecimento e em estabelecimentos diferentes, onde eles teriam que elaborar a solução do problema ou ate mesmo evitado esta situação.

Em outras atividades como curativos e centro cirúrgico após aulas expositivas e retirada de dúvidas os mesmos foram levados em grupos para os setores específicos e nos locais específicos tiveram oportunidade de realizar a atividade na prática havendo tempo disponível para retirada de duvidas e conversas dialogadas.

Outro diferencial foi demonstração de todos os produtos nas atividades que convinham como na de biossegurança, demonstração de EPIs, EPC e adornos. Na atividade de curativos, demonstração de todas as coberturas, atividade de esterilização, demonstração dos produtos e técnicas assépticas.

Os profissionais melhoraram após as capacitações que foram realizadas de diversas formas, aulas expositivas, práticas e rodas de conversa. Estratégias utilizadas como didática e tática para permanência e participação da equipe.

Os modelos assistenciais pautados pela integralidade devem adotar formas ampliadas de captação das necessidades de saúde, criando possibilidades para atender não somente aquilo que está programado, ou que é identificado como relevante pelos saberes tradicional como a epidemiologia. Os modelos de saúde integral devem dotar seus profissionais da capacidade ampliada de apreensão da realidade a partir de fontes diversas como a escuta aos usuários, o olhar sobre o território, as demandas do controle social e o planejamento participativo (MATTOS, 2008).

A formação dos profissionais de saúde é ponto fundamental para a reforma sanitária e implementação do SUS. Além da qualificação técnica é necessário que estes profissionais tenham acesso a saberes capazes de auxiliá-los no acolhimento das necessidades de saúde da população, possibilitando a interação trabalhador usuário em uma relação pautada pela responsabilização mútua e construção de autonomia (SANTOS; TENÓRIO, KICH, 2011).

\section{Conclusão}

Considera a educação permanente essencial, um apoio as atividades a serem desenvolvidas na prática da saúde, partindo do ponto que a mesma deve ser realizada de 
forma dinâmica, envolvente, técnica com envolvimento de grupos buscando melhorias no serviço e trabalhando a necessidade de cada um, ou seja focado na coletividade.

De acordo com toda descrição do trabalho pode se observar a realização das atividades segundo cronograma e estratégias planejadas, as atividades foram realmente realizadas de forma mensal a partir de março a agosto de 2015 com temáticas diversificadas, escolhidas pela equipe da instituição e trabalhadas em coletividade, mas com intuito de também afetar a individualidade de cada um presente em cada momento.

Segundo as atividades desenvolvidas foram trabalhados as temáticas como acolhimento em saúde, ética no serviço, profissionais, responsabilidades, técnicas de curativos e coberturas existentes e sua utilização, biossegurança, centro cirúrgico e esterilização.

Pode se observar o sucesso em cada atividade, a partir da participação de cada um, a melhoria no dia a dia de trabalho que é acompanhado pelas enfermeiras do serviço e a descrição da satisfação após cada atividade, onde os participantes tinham a oportunidade de descrever em folhas ofícios e entregar aos facilitadores.

Este trabalho de intervenção deixou seu legado na instituição com a melhoria no trabalho e a capacitação de cada funcionário no que pertencia a sua área, fazendo com que a procura da ouvidoria pelos usuários diminuíssem e um serviço de melhor qualidade fosse ofertado pela instituição.

Em suma todas as atividades foram realizadas de forma criteriosa e com muita responsabilidade, a assiduidade dos profissionais foi exemplar, vale ressaltar que em alguns dias de atividades havia funcionários de folga, fato que não impediam a presença dos mesmos

Conclui afirmando que a Educação Permanente em Saúde é uma estratégia valida e essencial nos serviços de saúde, para traspôs os resultados em forma de serviço de qualidade, podendo ofertar a comunidade a acessibilidade, integralidade, equidade fazendo do Sistema Único de Saúde um trabalho de responsabilidade e satisfação.

\section{Referências}

SANTOS,Cláudia Menezes; TENÓRIO „Flavia Priscila Souza; KICH, Francis Deon . Educação Permanente em Saúde no Estado de Sergipe Saberes e tecnologias para implantação de uma política. Livro do Aprendiz. $1^{\text {a }}$ Ed. Aracaju-SE 2011. 
BRASIL. Ministério da Saúde. Conselho Nacional de Saúde. Lei no 8.080, de 19 de setembro de 1990. Brasília, 2015. Site http://conselho.saude.gov.br/legislacao/lei8080.htm Acesso em 02 de outubro de 2015.

FLORES, Lisiane Melo Muniz ; ILHA, Neida Luiza Pelenz. Educação Continuada em Enfermagem. Disciplinarum Scientia. Série: Ciên. Biol. e da Saúde, Santa Maria, v.2, n.1, p.79-86, 2001.

BRASIL. Ministério da Saúde. Secretaria de Gestão do Trabalho e da Educação na Saúde Departamento de Gestão da Educação em Saúde Política Nacional de Educação Permanente em Saúde. Série B. Textos Básicos de Saúde. Série Pactos pela Saúde 2006, v. 9 Brasília - DF 2009.

GIL,Antonio Carlos. Como elaborar projetos de pesqauisa.Atlas, São Paulo, 2010.

MATTOS, Ruben Araújo. Os sentidos da integralidade: algumas reflexões acerca de valores que merecem ser defendidos. Disponível em: http://www.uefs.br/pepscentroleste/arquivos/artigos/os_sentidos_integralidade.pdf.> Acesso em: 25 jul. 2010.

\section{Como citar este artigo (Formato ABNT)}

ALENCAR, A.P.A.; FONSECA, F.L.A.; SILVA, M.C.S.; MARQUES, A.C.; LIRA, P.F.; FIGUEIREDO, C.M.; XAVIER, S.P.L. Educação Permanente: Estratégia Resolutiva na Enfermagem. Id on Line Revista Multidisciplinar e de Psicologia, Julho de 2016, vol.,10 n.30, Supl 1. p. 202-209. ISSN 1981-1179.

Recebido: 15/06/2016

Aceito: 20/06/2016 